\title{
IL7RA haplotype-associated alterations in cellular immune function and gene expression patterns in multiple sclerosis
}

\author{
Jäger, J ; Schulze, C ; Rösner, S ; Martin, R
}

\begin{abstract}
Interleukin-7 receptor alpha (IL7RA) is among the top listed candidate genes influencing the risk to develop multiple sclerosis (MS), an inflammatory demyelinating disease of the central nervous system. Soluble IL-7RA (sIL-7RA) protein and mRNA levels vary among the four common IL7RA haplotypes. Here we show and confirm that protective haplotype carriers have three times lower sIL-7RA serum levels than the other three haplotypes. High sIL-7RA concentrations significantly decrease IL-7-mediated STAT5 phosphorylation in CD4(+) T cells. Transcriptome analysis of unstimulated and stimulated CD4(+) T cells of MS patients carrying the different IL7RA haplotypes revealed complex and overlapping patterns in genes participating in cytokine signaling networks, apoptosis, cell cycle progression and cell differentiation. Our findings indicate that genetic variants of IL7RA result in haplotype-associated differential responsiveness to immunological stimuli that influence MS susceptibility not exclusively by varying levels of sIL-7RA.
\end{abstract}

DOI: https://doi.org/10.1038/gene.2013.40

Posted at the Zurich Open Repository and Archive, University of Zurich ZORA URL: https://doi.org/10.5167/uzh-85437

Journal Article

Accepted Version

Originally published at:

Jäger, J; Schulze, C; Rösner, S; Martin, R (2013). IL7RA haplotype-associated alterations in cellular immune function and gene expression patterns in multiple sclerosis. Genes and immunity, 14(7):453-461.

DOI: https://doi.org/10.1038/gene.2013.40 


\title{
IL7RA Haplotype-Associated Alterations in Cellular Immune Function and Gene Expression Patterns in Multiple Sclerosis
}

\author{
Jan Jäger ${ }^{1 \#}$, Christian Schulze ${ }^{2 \#}$, Sabine Rösner ${ }^{1,3}$, Roland Martin ${ }^{1,4^{*}}$
}

${ }^{1}$ Institute for Neuroimmunology and Clinical Multiple Sclerosis Research (inims), Centre for Molecular Neurobiology Hamburg (ZMNH), University Medical Centre Hamburg-Eppendorf, 20251 Hamburg, Germany

${ }^{2}$ Systems Biology, Centre for Molecular Neurobiology Hamburg (ZMNH), University Medical Centre Hamburg-Eppendorf, 20251 Hamburg, Germany

${ }^{3}$ Department of Neurology, University Medical Centre Hamburg-Eppendorf, 20246 Hamburg, Germany

${ }^{4}$ Neuroimmunology and Multiple Sclerosis Research, Department of Neurology, University Hospital Zurich, University of Zurich, Frauenklinikstraße 26, 8091 Zurich, Switzerland

\section{* Corresponding author:}

Roland Martin, MD

Neuroimmunology and Multiple Sclerosis Research,

Department of Neurology, University Hospital Zurich, University of Zurich

Frauenklinikstraße 26

8091 Zurich

Switzerland

Tel No: +41(0)44255 1125

Fax No: +41 (0)442554507

Email: roland.martin@usz.ch

\# J.Jäger and C.Schulze contributed equally to the study. 


\section{Abstract:}

Interleukin-7 receptor alpha (IL-7RA) is among the top listed candidate genes influencing the risk to develop multiple sclerosis (MS), an inflammatory demyelinating disease of the central nervous system. Soluble IL-7RA (sIL-7RA) protein and mRNA levels vary among the four common IL7RA haplotypes. Here we show and confirm that protective haplotype carriers have three times lower sIL-7RA serum levels than the other three haplotypes. High sIL-7RA concentrations significantly decrease interleukin-7-(IL-7) mediated STAT5 phosphorylation in $\mathrm{CD}^{+} \mathrm{T}$ cells. Transcriptome analysis of unstimulated and stimulated $\mathrm{CD}^{+} \mathrm{T}$ cells of MS patients carrying the different IL7RA haplotypes revealed complex and overlapping patterns in genes participating in cytokine signaling networks, apoptosis, cell cycle progression and cell differentiation. Our findings indicate that genetic variants of IL7RA result in haplotype-associated differential responsiveness to immunologic stimuli that influence MS susceptibility not exclusively by varying levels of sIL-7RA.

Keywords: interleukin-7 receptor; polymorphism; multiple sclerosis 


\section{Introduction}

Multiple sclerosis (MS) is an inflammatory demyelinating disease of the central nervous system (CNS). It affects mainly adults between 20 and 40 years of age ${ }^{1}$. The etiology of MS is still incompletely understood, but both a complex genetic trait and environmental factors contribute to disease expression ${ }^{2}$. At the level of individual genes the most important contribution to genetic risk stems from alleles in the human leukocyte antigen (HLA) class two region, namely the DR15 haplotype ${ }^{3}$. Genome-wide association studies (GWAS) have discovered until today more than 50 common genetic variants, single nucleotide polymorphisms (SNP), that contribute to MS susceptibility ${ }^{4,5}$, and it is expected that additional ones will be discovered in the near future. SNPs in the genomic region coding for the IL-7RA were the second set of confirmed MS risk alleles ${ }^{6-8}$.

Among the IL7RA SNPs the nonsynonymous SNP rs6897932 located in exon 6 in the IL7RA gene shows the strongest association with MS ${ }^{7,8}$. Several functional studies demonstrated that the risk " $C$ " allele leads to an increased skipping of exon 6 resulting in a decreased ratio of full length- to soluble IL-7RA-encoding mRNA and an increased amount of slL-7RA $(\mathrm{H} 6)^{7,9,10}$. While this was repeatedly shown it remains an open question why only one out of three haplotypes comprising rs6897932 is positively associated with MS. Four SNPs, rs1494555, rs6897932, rs987107 and rs987106, are sufficient to tag the four main haplotypes that arise from the IL7RA gene locus (Figure 1) ${ }^{7}$. Haplotype 1 is also referred to as the "risk" haplotype, because it is more prominent in MS patients compared to healthy donors (HD), whereas the opposite effect has been observed for haplotype 3 , which has therefore been termed "protective" haplotype.

Regarding the functional effects of IL7RA haplotypes beyond altered SIL-7RA levels previous studies have shown differences between haplotype 2 and non-haplotype 2 carriers with respect to the frequency of recent thymic emigrants ${ }^{11}$ and response of $C D 4^{+}$ lymphocytes to interferon beta ${ }^{12}$. The functional effects on $\mathrm{T}$ cell stimulation of haplotype 2 , which has not been found to related to MS risk, is thought to arise from differences in the genomic sequences within the promoter region of the IL7RA locus, which are also tagged by the SNPs used to define haplotypes.

In the context of MS and autoimmune diseases IL-7-mediated signaling likely acts through its involvement in lymphocyte development and/or homeostasis, but this may not be the only mechanism. IL-7 signaling through the IL-7R is indispensable for the development of human $T$ lymphocytes ${ }^{13}$ as demonstrated by the $X$-linked severe combined immunodeficiency (XSCID) phenotype of patients carrying a defect in the IL7RA. Given the central role of $\mathrm{T}$ cells in MS and other autoimmune diseases it is therefore reasonable to 
assume that IL-7/IL-7R interactions are functionally involved in their pathogenesis, and it is interesting that not only SNPs in IL7RA, but also in the cytokine IL-7 are associated with MS 14. The IL-7RA is mainly expressed by cells of the lymphoid lineage, but also by dendritic cells and monocytes ${ }^{15,16}$. IL-7, is produced by non-hematopoietic stromal- and epithelial cells, but to lesser amounts also by dendritic cells (DC) ${ }^{17}$. Binding of IL-7 leads to dimerization of the IL-7RA chain and the so-called common $\gamma$ chain $(\gamma \mathrm{C})$, which then form the high affinity IL-7R ${ }^{18}$. IL-7R shares its $\alpha$ chain with the thymic stromal lymphopoietin (TSLP) receptor, and the common $\gamma$ chain with the receptors for IL-2, IL-4, IL-9, IL-15 and IL-2 ${ }^{19}$. IL7R-mediated signaling activates the JAK/STAT and PI3K/AKT signaling pathways ${ }^{15,16}$. The homeostasis of naïve T lymphocytes is regulated by TCR and self-peptide MHC contact and the availability of pro-survival cytokines such as IL-7 ${ }^{16,18}$. Upon ligand engagement activatedas well as IL-7-stimulated T lymphocytes down-regulate the IL-7R, and since IL-7 is produced at relatively stable, but low levels by the abovementioned stromal cells, more IL-7 is then available for naïve and memory $T$ lymphocytes with the result that diverse repertoires of naïve and antigen-experienced memory $T$ cell populations are maintained ${ }^{15}$.

IL-7 also plays a role in other autoimmune diseases such as rheumatoid arthritis (RA) where it has been shown that IL-7 serum and synovial fluid concentrations are elevated in comparison to healthy donors and in addition are correlated with disease activity ${ }^{20}$. Hartgring and colleagues ${ }^{21}$ were able to demonstrate in an in vitro model that recombinant SIL-7RA reduces IL-7-mediated proliferation and production of interferon gamma (IFN- $\gamma$ ) of PBMCs. Interestingly, anti-IL-7RA antibody treatment of either mice with collagen-induced arthritis $(\mathrm{CIA})$ or experimental autoimmune encephalomyelitis $(\mathrm{EAE})$, led to reduced disease severity $^{22-24}$. The above data indicate that dysregulation of the IL-7/IL-7R system may contribute to disease pathogenesis and pathologic immune activation during autoimmune diseases. Its physiological roles include not only the functions in T lymphocyte generation and -homeostasis, but also coping with chronic viral infections ${ }^{25}$.

Despite robust confirmation of the associations of IL7RA SNPS with MS, the understanding of the possible functional contributions of IL7RA risk-conferring alleles to MS pathogenesis is still incomplete. The goals of the present study were to characterize immune cells of MS patients carrying risk- or protective IL7RA alleles with respect to membrane- and sIL-7RA expression, to examine how the increased SIL-7RA serum concentrations in risk allele carriers impact on IL-7/IL-7R functions, and if transcriptional profiling in individuals carrying the various IL7RA haplotypes leads to additional insight into their potential functional contribution to MS. 


\section{Results}

\section{Association of SNP rs6897932 with MS}

First, we analyzed the IL7RA allele, genotype, and haplotype distribution in a cohort of Northern German, Caucasian MS patients $(n=484)$ and healthy donors (HD) $(n=310)$. Four tagging SNPs (rs1494555, rs6897932, rs987107 and rs987106) were used to identify the four common IL7RA haplotypes. Due to the small sample size we did not expect to reach statistical significance. Surprisingly, however, this was the case for SNP rs6897932 (Table 1). The dominant model fit best to our data and confirmed an increased MS susceptibility for homozygous carriers of the "C" allele of the SNP rs6897932 $(p=0.03 ; O R=1.38$ [95\% confidence interval: $1.04-1.84]$ ). No significant association with disease could be detected for the other three SNPs. However, the genotype frequencies in the Hamburg cohort were similar to previously published reports ${ }^{7,26}$. As expected for a small cohort, no statistical significance was reached for the haplotype distribution (Table 2).

\section{mRNA- and Protein Expression Correlate with IL7RA Haplotype}

Previous studies ${ }^{9,10}$ demonstrated an influence of the IL7RA haplotype on the splicing resulting in an increased serum concentration of sIL-7RA. To determine whether or not we can confirm these effects, we analyzed the IL-7RA mRNA- as well as protein expression in haplotype 1-4-carrying homozygous MS patients. The relative expression of the full length $(\mathrm{H} 20)$, the soluble isoform missing exon $6(\mathrm{H} 6)$ and all common IL-7RA isoforms was determined by RT-PCR. Carriers of the protective haplotype had an increased ratio in the expression of membrane bound to soluble IL-7RA compared to carriers of the other haplotypes (Figure $2 \mathrm{~A}$ ). In contrast and consistent with previous results risk allele carriers showed a significantly reduced ratio, i.e. a relative increase of the soluble isoform and a slight reduction of the full-length isoform (Supplementary Material, Figure S1).

We then examined the IL-7RA serum concentration of homozygous MS patients of the four common IL7RA haplotypes by ELISA. Analysis of sIL-7RA protein expression is in accordance with the gene expression analysis. Patients carrying the protective haplotype had three times lower sIL-7RA serum concentrations compared to carriers of the other three haplotypes (Figure 2 B). MS patients heterozygous for the SNP rs6897932 had intermediate slL-7RA serum levels (Figure $2 \mathrm{~B}$ ). Therefore, consistent with the reports of Hoe et al. ${ }^{9}$ we demonstrate an allele dosage influence of SNP rs6897932 on the slL-7RA serum concentration. 


\section{Altered IL-7RA Surface Expression on Natural Killer Cells in Relation to the IL7RA Haplotype}

Next, we addressed if carriers of the risk- versus protective IL7RA haplotypes show differences in membrane expression of the receptors on different immune cells including $\mathrm{CD}^{+}$and $\mathrm{CD}^{+}$bulk $\mathrm{T}$ cells, the naïve- and memory populations of these two subsets, regulatory $T$ cells, several subpopulations of dendritic cells, and the two main populations of natural killer cells (CD56 ${ }^{\text {dim }}$ and CD56 ${ }^{\text {bright }}$ ). We hypothesized that the greatest differences might be observed between the risk- and the protective haplotypes because these varied most with respect to their putative effects on MS susceptibility. We found a significant, albeit small difference for CD56 bright $\mathrm{NK}$ cells (Figure 3). Risk haplotype carriers showed significantly lower IL-7RA surface expression compared to carriers of the protective haplotype $(p$-value=0.014). While there were consistent trends towards lower IL-7RA membrane expression of several immune cell populations of risk haplotype carriers, e.g. in naïve $\mathrm{CD} 4^{+} \mathrm{T}$ cells and Tregs, these did not reach statistical significance (Supplementary Material, Figure S2).

\section{sIL-7RA Inhibits STAT5 Phosphorylation}

Next, we questioned if the sIL-7RA levels interfere with $T$ cell function by influencing the availability of cytokine for binding to the membrane-bound IL-7R and hence IL-7Rmediated signaling. Previous studies have shown that SIL-7RA can bind IL-7 ${ }^{27,28}$. Although the affinity is lower ${ }^{29,30}$ than that of the membrane-bound form, the soluble IL-7RA (concentrations in risk haplotype carriers $50-185 \mathrm{ng} / \mathrm{ml}$ ) may be capable of influencing the availability of IL-7, due to the fact that it is available in the serum at an up to ninety thousandfold excess compared to the free cytokine IL-7 (levels 2-8 pg/ml). So far most studies used the recombinant IL-7RA (R\&D, 306-IR-050) or sIL-7RA produced by WI26VA4 cells, which shed the IL-7RA upon IL-7 stimulation, to analyze the effect of sIL-7RA on IL-7 signaling. Because the $\mathrm{H} 6$ isoform, which misses exon 6 , is the most common soluble IL-7RA isoform in human plasma, we decided not to use the full length $(\mathrm{H} 20)$ or shed form of the IL-7RA, but express the soluble form lacking exon 6 and generated in excess in risk allele carriers. We established an IL-7RA (isoform H6)-secreting HEK cell line. Using this sIL-7RA (H6) we analyzed the effect of sIL-7RA on IL-7R-mediated STAT5 phosphorylation in human CD4 ${ }^{+} \mathrm{T}$ lymphocytes by flow cytometry. We observed clear differences between CD4 ${ }^{+} \mathrm{T}$ lymphocytes stimulated in absence or presence of sIL-7RA with IL-7 (Figure 4 A-D). The strongest effect was observed for $\mathrm{CD} 4^{+}$cells stimulated with $10 \mathrm{pg} / \mathrm{mL} \mathrm{IL}-7$ in presence of slL-7RA (isoform $\mathrm{H6}$ ), resulting in a $50 \%$ reduced STAT5 phosphorylation. According to our data the strength of the observed effect correlates with the ratio between IL-7 and the sIL-7RA (Figure 4 A-D). 
These data suggest a functional relevance of the increased SIL-7RA concentrations in carriers of the risk haplotype.

\section{IL7RA Haplotype-Associated Gene Expression Patterns}

The above data demonstrate that carriers of haplotype 1, 2 and 4 have increased sIL7RA serum levels due to differential splicing. Hence, it is remarkable that only haplotype 1 carriers show an increased risk for MS. We therefore wanted to address next, if the IL7RA haplotypes differ in further aspects beside the sIL-7RA concentration. Since CD4 ${ }^{+} \mathrm{T}$ cells are particularly critical for $\mathrm{MS}^{1}$, we performed global gene expression profiling of $\mathrm{CD}^{+} \mathrm{T}$ cells from MS patients that were homozygous for one of the three haplotypes (Hap1, Hap2, Hap3). Due to a lack of patients carrying haplotype 4, which is also the rarest of all four haplotypes, we were not able to include this haplotype in the gene expression analysis. Purified $\mathrm{CD}^{+} \mathrm{T}$ cells were analyzed unstimulated and after challenge with IL-7 to specifically address the IL-7/IL-7R system. Concentrations of IL-7 and sampling times were chosen based on pilot experiments monitoring BCL2 as major up-regulated gene following IL-7RA stimulation (not shown). The experimental design aimed at comparing gene expression profiles in carriers of the three haplotypes at basal level and more importantly also at examining haplotype-specific effects following IL-7RA stimulation.

Resting condition: Expression levels in unstimulated cells showed a trend towards haplotype-specific differences when a fraction of genes selected solely by the criteria of showing minimal intra-group variance was inspected with multidimensional scaling (843 Ensembl gene IDs, Supplementary Material, Table S1 and Figure S3 A). Although no gene reached statistical significance applying the widely used multiple test correction (BenjaminiHochberg; two-group t-test as well as three-group F-tests) (Table 3, Supplementary Material, Table S1 and Figure S3 A) we noted that the gene list contains several spliceosomal RNAs and micro RNAs. This may indicate that the different haplotypes are associated with varying capacity to react to cytokine stimulus at the transcriptional level especially for the extent to which splicing occurs, but also at the translational level for an immediate response to stimuli

IL-7-stimulated condition: The treatment of purified CD4 ${ }^{+} \mathrm{T}$ cells with IL-7 induced a robust response with the majority of the most significantly responding genes being upregulated (203/225 genes, top $1 \%$ of significantly regulated genes corresponding to adjusted p-value $\leq 1.6 \mathrm{E}-5$, total gene number at 0.05 level 3276 ). When combining individual samples by stimulus irrespective of haplotype assignment, particularly strong effects were seen for BCL2, CISH, IRF4, CDK6, PPAP2A, CEACAM1, ENO1, PTGER2, SLC37A3, CTPS (all upregulated) and FAIM3, IL7R, GCNT4, ARHGAP9, SORL1, DPEP2, MPP7, TMEM71, 
GIMAP2, ST6GALNAC2 (all down-regulated) (Table 4, Supplementary Material, Table S2 and Figure S3 B). For some of these genes a direct link to the JAK/STAT pathway, which is employed as the IL-7/IL-7R signaling pathway, is already known or may be inferred from analysis of STAT5-occupied DNA sites (CISH, BCL2, CDK6, CEACAM1, CTPS, GCNT4, PPAP2A, PTGER2, ST6GALNAC2, MPP7) ${ }^{31}$.

Analyzing individual responses considering the most stable signals for multidimensional scaling already showed grouping of individuals according to haplotypes (Figure $5 \mathrm{~A}$ ). Of note, this grouping was obtained from an exploratory data analysis with unsupervised clustering, i.e. blind to haplotype assignment, and cannot be accounted for by SNP rs6897932 alone. Further inspection made clear that a considerable number of genes that changed in expression after IL-7 stimulus did not show concordant changes or differed in effect size between haplotypes (Supplementary Material, Table S3). Given the complex and overlapping participation of MS-associated genes in several signaling pathways we applied an unsupervised clustering technique (nonnegative matrix factorization, NMF) in order to extract haplotype-specific features from our high-dimensional gene expression data. NMF assigns weights to genes from a common input list according to their discriminatory power, which allows to distinguish a given group from the remaining ones and hereby creates groupspecific profiles (metagenes).

We considered genes showing robust IL-7 responses (adjusted p-value $\leq 0.05$ ) in combination with differences in response between haplotypes ( $F$ test, $p$-value $\leq 0.05,209$ genes, see Methods, Supplementary Material, Table S3) for NMF and observed a highly reproducible clustering of individuals as judged by the reported cophenetic coefficient (peaking at factorization rank $\mathrm{k}=3$ ) almost independent of the applied algorithm (Supplementary Material, Figure S4 A-D). The IL-7 response signals from a combination of top-ranked metagene members clearly show an overlapping pattern of differential reactivity. We note that haplotype 1 and 2, both comprising the rs6897932 risk allele, differ in their response to a considerable amount (Figure 5 B, Supplementary Material, Table S3).

In order to assess whether the top metagene-contributing genes are related to a specific signaling pathway and biologically plausible in the context of IL-7/IL-7R signaling, we queried the Reactome database. IL7, IL7R, IL2RB, KRAS, IFNGR2, and SUMO1 all participate in cytokine signaling in immune system networks (Reactome ID 75790, Figure 5 C) and sub-networks. Combining this information with interaction data from STRING (confidence score $\geq 0.7$ ) identified even more genes from the input list to be associated with cytokine signaling. How and to which extent differential responses of those genes and potential additional partners might shape haplotype-associated responses is not clear, but it 
appears that despite the limited sample size the obtained interconnections indeed relate to a genome-defined response pattern to immunologic stimuli. Of note, three cytokines and cytokine receptors, i.e. IL-7, IL-7R and IL-2R that have been associated with MS risk appear as metagenes in this pathway (Figure $5 \mathrm{C}$ ). 


\section{Discussion}

IL-7 is a critical factor for regulating T lymphocyte development and -homeostasis ${ }^{32}$. The IL-7/IL-7R system has been described to play a role in MS as well as in other autoimmune diseases such as type 1 diabetes, rheumatoid arthritis and inflammatory bowel disease ${ }^{24,33,34}$. There is compelling evidence from animal models of these diseases that blocking of the IL-7RA leads to an amelioration of the disease course by modulating inflammatory processes ${ }^{22-24,33,34}$. For MS, we and others ${ }^{9}$ could demonstrate highly significant differences in the amount of sIL-7RA between the haplotypes. Carriers of the risk haplotype showed up to three times higher sIL-7RA serum concentrations compared to carriers of the protective haplotype. The observation, that sIL-7RA is capable of reducing IL7-mediated STAT5 phosphorylation in $\mathrm{CD}^{+} \mathrm{T}$ cells is in line with data of Crawley et al. ${ }^{28}$, who demonstrated that SIL-7RA reduces IL-7-mediated signaling in $\mathrm{CD}^{+} \mathrm{T}$ cells, even though these authors did not use the sIL-7RA $(\mathrm{H} 6)$, which is generated by differential splicing in MS risk haplotype carriers. Besides the JAK/STAT pathway, IL-7 also activates signaling pathways that influence cell metabolism as well as proliferative capacity ${ }^{35}$. Hence, the risk haplotype could result in reduced lymphocyte proliferation due to an inhibitory effect of the elevated sIL-7RA levels on IL-7-mediated signaling. An alternative interpretation is that the vast excess of sIL-7RA may serve as a sink or reservoir of IL-7, which could be released under conditions of for example lowered $\mathrm{pH}$, which may occur in a local inflammatory environment. These scenarios are not mutually exclusive but may rather be relevant in different contexts such as homeostatic versus immune-activating conditions. While our data do not exclude the latter hypothesis that increased sIL-7RA concentrations act immuneactivating rather than compromising homeostatic turnover, the inhibition of STAT5 signaling and reduction of proliferation by sIL-7RA ${ }^{21}$ argue for the latter.

Autoimmunity involves breakdown of immune tolerance and among other factors an increased proliferation of autoreactive $T$ lymphocytes. Thus, the increased SIL-7RA serum concentration and the reduced proliferation seem to contradict an increased risk of haplotype 1 carriers for MS. However, the immunomodulatory capacity of the excess of sIL-7RA might increase MS susceptibility in an indirect way. A recent study by Pellegrini et al. ${ }^{25}$ demonstrated that IL-7 treatment reinvigorates the immune response and improves protection against chronic viral infections. Furthermore, treatment with recombinant IL-7 promotes $\mathrm{T}$ cell recovery after allogeneic stem cell transplantation ${ }^{36}$ and also supports the elimination of JC polyoma virus in patients with progressive multifocal leukoencephalopathy (PML), an opportunistic and often fatal infection of the brain in immunocompromised individuals (Sospedra et al., unpublished). One possibility is that the lower bioavailability of IL-7 in carriers of the risk haplotype caused by sIL-7RA sequestration of IL-7, increases the 
susceptibility to viral infection. Since viral infections are known to precede MS relapses and considering that EBV is the most important environmental risk factor of $\mathrm{MS}^{37}$, the increased levels of sIL-7RA and lower IL-7 bioavailability may lead to an at least subtle compromise in controlling viral infections, which then indirectly might contribute to onset of MS and/or occurrence of relapses/perpetuation once it has begun. This is supported by recent findings that the MS risk-conferring SNP rs6897932 is among three SNPs that confer poor outcome in HIV infection ${ }^{38}$. Another line of supporting evidence is the reduced expression of IL-7RA on CD56 ${ }^{\text {bright }}$ NK cells, and therefore possibly compromises in generating or maintaining this subset of NK cells. CD56 bright $\mathrm{NK}$ cells are not only important for the control of latent/persistent viral infections ${ }^{39}$, but also have immunoregulatory properties ${ }^{40}$. CD56 ${ }^{\text {bright }}$ NK cells are reduced in numbers and compromised in function in MS patients ${ }^{41}$, and treatment with the anti-CD25 monoclonal antibody daclizumab leads to significant expansion of CD56 bright $\mathrm{NK}$ cells, resulting in a decrease of inflammatory disease activity and of MS relapses ${ }^{40,42}$.

When considering the influence of single quantitative trait loci (QTLs) like the MS riskassociated IL7RA SNP on a complex disease like MS they appear to contribute only in a very minor way to MS risk, and their disease association could only be detected by comparing large numbers of patients and controls ${ }^{5}$. One therefore expects that such QTLs act in concert with others and influence functional pathways that translate into increased proinflammatory cytokine secretion, $T$ cell activation and other immune mechanisms that functionally contribute to a disease like MS. Since it is very difficult to dissect such interactions in genetically highly heterogeneous individuals, it is not surprising that with few exceptions ${ }^{43}$ very little is known about the functional role of MS risk alleles. Also, one would not expect strong signals, e.g. with respect to an important aspect of immune function, in the context of common variants as this most likely would result in a readily observable phenotype. From a systems perspective however, it is conceivable, that even small differences in amount of sIL-7RA protein, bioavailability of IL-7 and/or transcriptional activity defined by IL7RA haplotypes shape the individual immune response and eventually sum up to discernible amounts. At the typical age of onset around 30 years, MS patients have undergone numerous immunologic challenges. It is therefore highly interesting to note that Lundström et al. ${ }^{44}$ find differences in IL-7 levels between rs6897932 genotypes in MS patients. Considering these aspects our data on the complex pattern of differential response in $\mathrm{CD}^{+} \mathrm{T}$ cells in IL7RA haplotype homozygous individuals are not too unexpected and suggest that MS individuals differ in their response to immunologic stimuli. The application of conservative statistical significance thresholds will in these instances tend to underestimate biologically relevant information. 
As presented above, among the top induced changes in gene expression following stimulation of $\mathrm{CD}^{+}$cells with IL-7 there were no major haplotype-specific differences. In brief, the signal given by IL-7/IL-7R affects regulation of apoptosis, cell cycle progression, and differentiation (up-regulation of CISH, BCL2, CDK6, CTPS, IRF4 and ENO1, downregulation of IL7R, FAIM3, GIMAP2, TMEM71). In addition, substantial remodeling of cell-cell and cell-substrate interactions, adhesive properties, and cellular skeleton is triggered (CEACAM1, SORL1, MPP7, ARHGAP9, ST6GALNAC2). Interestingly, genes involved in lipid uptake and metabolism also show considerable expression level changes (PTGER2, DPEP2). Of note, PPAP2A (phosphatidic acid phosphatase type 2A) exhibits high phosphatase activity toward FTY720 phosphate, the active form of the immunomodulating agent FTY720 (Fingolimod), which is an approved drug for the treatment of MS ${ }^{45}$. Among the many affected genes the IL-7 response of a limited number of these differs between haplotypes and can be used to derive transcriptional signatures ('metagenes') indicating variable processing of cytokine signals. Interestingly, genes in the first-line response to pathogens are top metagene-defining features. These include among others IFNGR2, the receptor for probably the most important pro-inflammatory cytokine in several autoimmune diseases including MS, TLR2, which plays a fundamental role in pathogen recognition including also viruses like EBV, CX3CR1, a chemokine acting as a coreceptor for HIV-1, and ALOX5, a lipoxygenase gene family member, for which over-expression in MS, both in relapse and remission, was reported recently. The transcription factor XBP1, which regulates MHC class II genes, acts as viral transactivator and also responds to endoplasmatic reticulum stress, was placed among the top metagenes. The ability to respond to environmental stimuli is also defined by metabolic fitness and sensitivity to apoptosis of immune cells. Reflecting this, NDUFA2 and NDUFV2, which participate in mitochondrial electron transport chain assembly, came up in our network analysis. Interestingly, it has been proposed that mitochondrial proteins, and especially haplotypic variants, may also influence progression of viral diseases like AIDS ${ }^{46}$. Of interest for a neurological disease like MS, compromise of mitochondrial function and metabolic compromise of neurons have been implicated in neurodegenerative processes ${ }^{47}$. Although speculative at this point, the expression of IL-7RA in the central nervous system ${ }^{48,49}$ may indicate that it exerts an influence not only in the immune system, but also in the brain.

On the basis of our findings, we hypothesize that the IL7RA risk haplotype increases the susceptibility for viral infection, thereby enhancing the risk for MS. However, MS is a disease with a complex genetic trait, in which the IL7RA is only one piece in the extending puzzle of MS genetics. It is most likely that the IL7RA acts in concert with other genes to trigger MS. Here we show that by combining genetic information, specific cytokine stimulus, gene expression-, and network analysis one can gain insight into the complex interactions of 
sets of haplotype-specific metagenes and involved signaling pathways, which together suggest an influence of IL7RA haplotypes not only on immune mechanisms, but possibly also metabolic function and an involvement in neurodegenerative aspects of MS. This approach is promising in addressing the functional implications of individual polymorphisms with small effects found in many genome-wide association studies and expression quantitative trait loci studies on autoimmune diseases. 


\section{Methods}

\section{Subject and Sample Collection}

Patient material was collected from 484 MS patients $(29.8 \%$ males, $70.2 \%$ female, with a mean age of 45 years and a mean EDSS of 3.3) and 310 healthy donors ( $50 \%$ male, $50 \%$ female, with a mean age of 37 years) from the Hamburg cohort available at University Medical Center Hamburg-Eppendorf. Informed consent was obtained from all MS patients and healthy donors. All patients had a clinically defined MS according to McDonald criteria ${ }^{50}$. Peripheral blood was collected in ETDA tubes (Sarstedt, Nümbrecht, Germany) and serum was collected in serum monocyvettes (Sarstedt, Nümbrecht, Germany).

\section{Cell Isolation}

PBMCs were isolated by Ficoll (PAA, Pasching, Austria) gradient centrifugation, washed twice, resuspended in RPMI 1640 supplemented with 10\% DMSO and 20\% FCS and cryopreserved. $C D 4^{+} T$ cells were enriched from freshly isolated PBMCs by using the BD enrichment set DM (BD Biosciences, New Jersey, USA) following manufacturer's protocol achieving a purity of at least $95 \%$, verified by flow cytometry.

\section{Genotyping}

Genomic DNA was isolated from whole blood. Cells were lysed (1.6 M sucrose, 5\% v/V Triton X-100, $25 \mathrm{mM} \mathrm{MgCl}{ }_{2}$ and $60 \mathrm{mM}$ Tris-HCl pH 7.5), centrifuged (1950 g), the pellet resolved in $\mathrm{H}_{2} \mathrm{O}$ supplemented with proteinase $\mathrm{K}$ and $20 \%$ SDS, incubated over night at $37^{\circ} \mathrm{C}$, centrifuged $(3000 \mathrm{~g})$ and precipitated with $\mathrm{NaCl}(6 \mathrm{M})$. After centrifugation (3000 g), the DNA was washed with $100 \%$ and $70 \%$ ethanol. Four SNPs in the IL7RA gene (rs987106, rs987107, rs1494555, rs6897932) were analyzed using predesigned TaqMan probes (Applied Biosystems California, USA). The reaction was performed in an Applied Biosystems 7900 Real-Time PCR system.

\section{Association Analysis}

Genotype information was analyzed with Haploview $4.2^{51}$ and PLINK v1.07 (Shaun Purcell; http://pngu.mgh.harvard.edu/purcell/plink/). None of the obtained genotype distributions did show significant deviation from Hardy-Weinberg equilibrium. 


\section{Gene Expression Analysis of Different IL-7RA Isoforms}

RNA was isolated form PBMC-pellets using RNeasy kit (Qiagen, Hilden, Germany). cDNA synthesis was performed with Fermentas RevertAid $\mathrm{H}$ Minus first strand cDNA synthesis kit (Fermentas, St. Leon-Rot, Germany). Three pre-designed TaqMan (Applied Biosystems, California, USA) probes (HS_00902334_m1, HS_00904815_m1, Hs_00902337_m1) were used to monitor membrane bound (H20), the soluble (H6) IL-7RA isoform, and the total amount of IL-7RA encoding mRNA. The quantitative PCR reaction was performed in duplicates in an Applied Biosystems 7900 Real-Time PCR system.

\section{Quantification of sIL-7RA}

IL-7RA serum concentrations were determined by sandwich ELISA with capture antibody (MAB306), detection antibody (BAF306), HRP-conjugated streptavidin (DY998) and substrate (DY997), all from R\&D (Minnesota, USA). Serum samples were diluted 1/20 using PBS supplemented with $10 \%$ FCS. Microtiter plates were analyzed using a Perkin-Elmer (Massachusetts, USA) multilabel plate reader.

\section{Cell Surface Expression of IL-7RA on Immune Cell Populations}

Quantification of IL-7RA surface expression was performed with frozen PBMCs using flow cytometry (LSRII, BD Biosciences, New Jersey, USA). In all stainings duplicates were eliminated. $\mathrm{CD}^{+}$and $\mathrm{CD} 8^{+}$T-cells subtypes were identified by labeling the cells with antibodies specific for CD4 (Caltag Laboratories), CD8 (BioLegend, San Diego, USA), CD27 (BD Biosciences, New Jersey, USA), CD45RO (BioLegend, San Diego, USA) and CD127 (Beckman and Coulter, Indianapolis, USA). IL-7RA expression on dendritic cells was analyzed by eliminating CD14 (BD Biosciences, New Jersey, USA) and CD19 (BD Biosciences, New Jersey, USA) positive cells and gating on CD1c, CD141 or CD303 (all from Miltenyi Biotec, Bergisch Gladbach, Germany) positive cells. Natural killer (NK) cells were subdivided into CD56 ${ }^{\text {dim }}$ and CD56 ${ }^{\text {bright }}$ by using antibodies specific for CD3 (eBioscience, San Diego, USA), CD16 (BioLegend, San Diego, USA), CD56 (eBioscience, San Diego, USA) and CD127 (BioLegend, San Diego, USA). Tregs were defined as CD3, CD4 and FoxP3 positive and CD25 high expressing cells. The following antibodies were used CD3 (BD Biosciences, New Jersey, USA), CD4 and CD127 (BioLegend, San Diego, USA), CD25 (eBioscience, San Diego, USA) and intracellular FoxP3 and FoxP3 staining buffer set (Miltenyi Biotec, Bergisch Gladbach, Germany). The stainings were performed according to manufacturers' protocols. 


\section{Cloning and Stable Expression of Soluble IL-7RA}

Human cDNA encoding the soluble IL-7RA (H6; AK301220) was amplified in two

steps. First the regions encoded by exon 1-5 (forward-primer: 5'AATGACAATTCTAGGTACAACTTTTGGCATGGTTTTTTC; reverse-primer: CGATAGGCTTAATCCTGAGCTATTATTG) and 7-8 (forward-primer: $5^{\prime}-$ CAATA ATAGCTCAGGATTAAGCCTATCG; reverse-primer: 5'-CTCACTTTTCTTGGT TTCTTACAAAGATGTTC) were amplified and in a second step the two constructs were combined and modified with EcoRI and Xhol binding-sites (forward-primer: 5'GTAGAATTCGCCTCCATGACAATTCTAGGTACAACTTTTGGCATGGTTTT, reverse-primer: 5'-GACTCGAGCTCACTTTTCTTGGTTTCTTAC). The construct was cloned into pcDNA3.1 (Invitrogen, California, USA) and transformed into E. coli strain Top10F-.

Stable transfection of HEK293 cells was performed using Lipofectamine 2000 (Invitrogen, California, USA) according to the manufacturer's protocol. In short: HEK cells with a density of approximately $80 \%$ were transfected with $3 \mu \mathrm{g}$ linearised DNA and grown under selection conditions in DMEM (Invitrogen, California, USA) supplemented with 10\% FCS and 0.8 $\mathrm{mg} / \mathrm{ml} \mathrm{G} 418$ (PAA, Pasching, Austria). The efficiency of the transfected HEK cells to secrete IL-7RA was determined by ELISA (see above).

\section{STAT5 Phosphorylation}

The phosphorylation state of STAT5 was measured by flow cytometry. Freshly isolated $\mathrm{CD}^{+} \mathrm{T}$ cells were pre-incubated for $2 \mathrm{~h}$ at $37^{\circ} \mathrm{C}$ in RPMI 1640 Glutamax (PAA, Pasching, Austria) supplemented with 10\% FCS and then stimulated with the as well preincubated stimulus for $15 \mathrm{~min}$. The following components were used for stimulation: IL-7 (Sigma Aldrich, Missouri, USA), concentrated HEK-cell supernatant and concentrated supernatant of a HEK-cell line producing soluble IL-7RA. Directly after stimulation the cells were fixed and permeabilized by using Cytofix Buffer and 1x Perm Buffer III (both from BD Biosciences, New Jersey, USA). Phospho-STAT5 (anti-STAT5-P (Y694), BD Biosciences, New Jersey, USA) was measured by flow cytometry.

\section{Transcriptome Analysis}

Transcriptomes from $\mathrm{CD}^{+} \mathrm{T}$ cells from patients with haplotype $1(\mathrm{n}=3)$, haplotype 2 $(n=2)$, and haplotype $3(n=2)$ were recorded using Affymetrix human gene array chips (GeneChip Human Gene 1.0 ST Array) hybridized at Transkriptomanalyselabor, University of

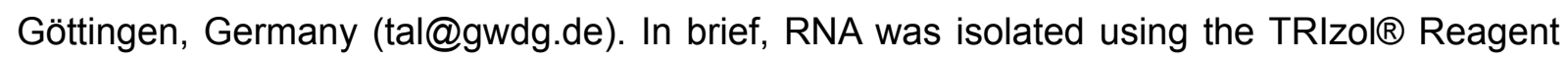
(Invitrogen) and checked for quantity, purity and integrity using the Agilent 2100 bioanalyzer 
(Agilent Technologies). Microarray hybridization and data extraction was done according to the manufacturer's specifications.

Raw microarray data were background corrected and normalized by applying robust multichip average and quantile normalization. Processed probe expression values were condensed into Affymetrix transcript clusters and filtered for genes containing Entrez gene identifier. Differential expression was calculated via fitting a linear model with moderated $t-$ statistics to groups defined by haplotype, treatment, and combinations of haplotype and treatment. All calculations were done in R/Bioconductor ${ }^{52}$. For initial exploratory data analysis, expression levels of unstimulated cells were inspected for genes (only autosomal location) that showed small median absolute deviation (lower third calculated per haplotype group) with a reasonable minimal expression (threshold set to 4.0). The resulting set corresponding to 843 Ensembl gene IDs was subjected to non-metric multidimensional scaling (Sammon's Non-Linear Mapping) and plotted. Haplotype specific effects were inspected in a similar way. For detailed analysis of haplotype-associated signatures we used genes showing robust response to IL-7 treatment in at least one of the haplotype groups (Benjamini-Hochberg adjusted $p$-value $\leq 0.05$ ) which at the same time differed between haplotypes (F-test, $p$-value $\leq 0.05$ ). Data with high intra group variation taken as median absolute deviation $\geq 0.5$ were removed. Nonnegative matrix factorization (NMF) on the resulting 207 differentially responding genes with autosomal location as input (completed with data for IL-7 and IL-7RA) was used to extract subsets of haplotype-specific genes ${ }^{53}$. In $\mathrm{NMF}$, the gene expression levels are treated as matrix $\mathrm{X}$ consisting of $\mathrm{N}$ genes in $\mathrm{M}$ samples that is factorized according to $\mathrm{X}=\mathrm{W} \times \mathrm{H}$ into a metagene matrix $\mathrm{W}$ and a metagene expression profile (meta experiment) matrix $\mathrm{H}$. Columns of $\mathrm{W}$ hold the contribution of genes to a metagene, and columns of $\mathrm{H}$ describe the metagene composition corresponding to each sample. The optimal rank $\mathrm{k}$ (number of extracted basis components) was inferred from plotting the cophenetic correlation coefficient versus rank and was set to three. Initial tests showed that rank $k=3$ gave optimal clustering and that nonsmooth NMF performed best (Supplementary Material, Figure S4). The consensus matrix (average connectivity between metagenes and samples) obtained from using a NMF algorithm with random initialization after 1000 runs showed stable clustering of samples ${ }^{54}$. The interaction network of top metagenes was assembled in Cytoscape using Reactome and STRING databases. STRING-based associations of further genes (first neighbors only) were considered with combined score $\geq 0.7$ (high confidence).

Data are available through Gene Expression Omnibus (GSE37584). 


\section{Acknowledgements}

We thank Dr T. Eiermann and Dr T. Binder, Department of Transfusion Medicine, University Medical Centre Hamburg-Eppendorf, for generous supply of healthy donor DNA samples and Brenda Reinhart, PhD, Neuroimmunology and MS Research, and Michal Okoniewski, PhD, Neuroimmunology and MS Research and Functional Genomics Center Zurich for helpful comments and carefully reading the manuscript.

\section{Funding}

The Institute for Neuroimmunology and Clinical Multiple Sclerosis Research (inims), was supported by a five-year grant of the Gemeinnützige Hertie Stiftung; and this project was supported by the Deutsche Forschungsgemeinschaft (MA 965/9-1). The Section of Neuroimmunology and MS Research is supported by the Clinical Research Priority Program MS (CRPP ${ }^{\mathrm{MS}}$ ) of the University Zurich.

\section{Conflict of Interest Statement}

The authors have no financial conflict of interest. R. Martin is co-inventor on a patent for the combined use of recombinant IL-7 and vaccination with JC polyoma virus capsid protein VP1 to treat progressive multifocal leukoencephalopathy (PML).

\section{Supplementary Information}

Supplementary Information accompanies the paper on Genes and Immunity website (http://www.nature.com/gene) 


\section{References}

1 Sospedra M, Martin R. Immunology of multiple sclerosis. Annual review of immunology 2005; 23: 683-747.

2 Oksenberg JR, Baranzini SE, Sawcer S, Hauser SL. The genetics of multiple sclerosis: SNPs to pathways to pathogenesis. Nature reviews Genetics 2008; 9: 51626.

3 Olerup O, Hillert J. HLA class II-associated genetic susceptibility in multiple sclerosis: a critical evaluation. Tissue antigens 1991; 38: 1-15.

$4 \quad$ Kemppinen A, Sawcer S, Compston A. Genome-wide association studies in multiple sclerosis: lessons and future prospects. Briefings in functional genomics 2011; 10: $61-$ 70.

5 Sawcer S, Hellenthal G, Pirinen M, Spencer CC a., Patsopoulos N a., Moutsianas L, et al. Genetic risk and a primary role for cell-mediated immune mechanisms in multiple sclerosis. Nature 2011; 476: 214-9.

6 Lundmark F, Duvefelt K, lacobaeus E, Kockum I, Wallström E, Khademi M, et al. Variation in interleukin 7 receptor alpha chain (IL7R) influences risk of multiple sclerosis. Nature genetics 2007; 39: 1108-13.

7 Gregory SG, Schmidt S, Seth P, Oksenberg JR, Hart J, Prokop A, et al. Interleukin 7 receptor alpha chain (IL7R) shows allelic and functional association with multiple sclerosis. Nature Genetics 2007; 39: 1083-91.

8 The International Multiple Sclerosis Genetics Consortium, Hafler DA, Compston A, Sawcer S, Lander ES, Daly MJ, et al. Risk alleles for multiple sclerosis identified by a genomewide study. The New England journal of medicine 2007; 357: 851-62.

9 Hoe E, McKay FC, Schibeci SD, Gandhi K, Heard RN, Stewart GJ, et al. Functionally significant differences in expression of disease-associated IL-7 receptor alpha haplotypes in CD4 T cells and dendritic cells. Journal of immunology (Baltimore, Md $\square$ : 1950) 2010; 184: 2512-7.

10 McKay FC, Swain LI, Schibeci SD, Rubio JP, Kilpatrick TJ, Heard RN, et al. Haplotypes of the interleukin 7 receptor alpha gene are correlated with altered expression in whole blood cells in multiple sclerosis. Genes and Immunity 2008; 9: 16.

11 Broux B, Hellings N, Venken K, Rummens J-L, Hensen K, Van Wijmeersch B, et al. Haplotype 4 of the multiple sclerosis-associated interleukin-7 receptor alpha gene influences the frequency of recent thymic emigrants. Genes and Immunity 2010; 11: 326-33.

12 Hoe E, McKay F, Schibeci S, Heard R, Stewart G, Booth D. Interleukin 7 receptor alpha chain haplotypes vary in their influence on multiple sclerosis susceptibility and response to interferon Beta. Journal of interferon \& cytokine research $\square$ : the official journal of the International Society for Interferon and Cytokine Research 2010; 30: 291-8. 

critical growth factor in early human T-cell development. Blood 1996; 88: 4239-45.

14 Zuvich RL, McCauley JL, Oksenberg JR, Sawcer SJ, De Jager PL, Aubin C, et al. Genetic variation in the IL7RA/IL7 pathway increases multiple sclerosis susceptibility. Human Genetics 2010; 127: 525-35.

15 Mackall CL, Fry TJ, Gress RE. Harnessing the biology of IL-7 for therapeutic application. Nature reviews Immunology 2011; 11: 330-42.

16 Palmer MJ, Mahajan VS, Trajman LC, Irvine DJ, Lauffenburger D a, Chen J. Interleukin-7 receptor signaling network: an integrated systems perspective. Cellular \& molecular immunology 2008; 5: 79-89.

17 Sorg R V, McLellan AD, Hock BD, Fearnley DB, Hart DN. Human dendritic cells express functional interleukin-7. Immunobiology 1998; 198: 514-26.

18 Mazzucchelli R, Durum SK. Interleukin-7 receptor expression: intelligent design. Nature reviews Immunology 2007; 7: 144-54.

19 Rochman Y, Spolski R, Leonard WJ. New insights into the regulation of T cells by gamma(c) family cytokines. Nature reviews Immunology 2009; 9: 480-90.

20 Van Roon JAG, Glaudemans KAFM, Bijlsma JWJ, Lafeber FPJG. Interleukin 7 stimulates tumour necrosis factor alpha and Th1 cytokine production in joints of patients with rheumatoid arthritis. Annals of the rheumatic diseases 2003; 62: 113-9.

21 Hartgring S a Y, Van Roon J a G, Wenting-van Wijk M, Jacobs KMG, Jahangier ZN, Willis CR, et al. Elevated expression of interleukin-7 receptor in inflamed joints mediates interleukin-7-induced immune activation in rheumatoid arthritis. Arthritis and rheumatism 2009; 60: 2595-605.

22 Lee L-F, Axtell R, Tu GH, Logronio K, Dilley J, Yu J, et al. IL-7 Promotes TH1 Development and Serum IL-7 Predicts Clinical Response to Interferon- in Multiple Sclerosis. Science Translational Medicine 2011; 3: 93ra68-93ra68.

23 Ashbaugh JJ, Brambilla R, Karmally S a, Cabello C, Malek TR, Bethea JR. IL7Ra Contributes to Experimental Autoimmune Encephalomyelitis through Altered T Cell Responses and Nonhematopoietic Cell Lineages. Journal of immunology (Baltimore, Md $\square:$ 1950) 2013; 190: 4525-34.

24 Hartgring SAY, Willis CR, Alcorn D, Nelson LJ, Bijlsma JWJ, Lafeber FPJG, et al. Blockade of the interleukin-7 receptor inhibits collagen-induced arthritis and is associated with reduction of T cell activity and proinflammatory mediators. Arthritis and rheumatism 2010; 62: 2716-25.

25 Pellegrini M, Calzascia T, Toe JG, Preston SP, Lin AE, Elford AR, et al. IL-7 engages multiple mechanisms to overcome chronic viral infection and limit organ pathology. Cell 2011; 144: 601-13.

26 Weber F, Fontaine B, Cournu-Rebeix I, Kroner a, Knop M, Lutz S, et al. IL2RA and IL7RA genes confer susceptibility for multiple sclerosis in two independent European populations. Genes and immunity 2008; 9: 259-63. 
27 Goodwin RG, Friend D, Ziegler SF, Jerzy R, Falk B a, Gimpel S, et al. Cloning of the human and murine interleukin-7 receptors: demonstration of a soluble form and homology to a new receptor superfamily. Cell 1990; 60: 941-51.

28 Crawley AM, Faucher S, Angel JB. Soluble IL-7R alpha (sCD127) inhibits IL-7 activity and is increased in HIV infection. Journal of immunology (Baltimore, Md $\square$ : 1950) 2010; 184: 4679-87.

29 Blom-Potar M-C, Bugault F, Lambotte O, Delfraissy J-F, Thèze J. Soluble IL-7Ralpha (sCD127) and measurement of IL-7 in the plasma of HIV patients. Journal of acquired immune deficiency syndromes (1999) 2009; 51: 104-5.

30 Rose T, Lambotte O, Pallier C, Delfraissy J-F, Colle J-H. Identification and biochemical characterization of human plasma soluble IL-7R: lower concentrations in HIV-1-infected patients. Journal of immunology (Baltimore, Md $\square: 1950$ ) 2009; 182 : 7389-97.

31 Zhu B, Kang K, Yu J, Chen W. Genome-wide analyses reveal the extent of opportunistic STAT5 binding that does not yield transcriptional activation of neighboring genes. Nucleic acids research 2012; 40: 4461-72.

32 Carrette F, Surh CD. IL-7 signaling and CD127 receptor regulation in the control of T cell homeostasis. Seminars in immunology 2012; 24: 209-17.

33 Lee L, Logronio K, Tu GH, Zhai W, Ni I, Mei L, et al. Anti-IL-7 receptor-a reverses established type 1 diabetes in nonobese diabetic mice by modulating effector T-cell function. Proceedings of the National Academy of Sciences of the United States of America 2012; 109: 12674-9.

34 Willis CR, Seamons A, Maxwell J, Treuting PM, Nelson L, Chen G, et al. Interleukin-7 receptor blockade suppresses adaptive and innate inflammatory responses in experimental colitis. Journal of inflammation (London, England) 2012; 9: 39.

35 Kittipatarin C, Khaled AR. Interlinking interleukin-7. Cytokine 2007; 39: 75-83.

36 Perales M-A, Goldberg JD, Yuan J, Koehne G, Lechner L, Papadopoulos EB, et al. Recombinant human interleukin-7 (CYT107) promotes T-cell recovery after allogeneic stem cell transplantation. Blood 2012; 120: 4882-91.

37 Wandinger K, Jabs W, Siekhaus A, Bubel S, Trillenberg P, Wagner $\mathrm{H}$, et al. Association between clinical disease activity and Epstein-Barr virus reactivation in MS. Neurology 2000; 55: 178-84.

38 Limou S, Melica G, Coulonges C, Lelièvre J-D, Do H, McGinn S, et al. Identification of IL7RA risk alleles for rapid progression during HIV-1 infection: a comprehensive study in the GRIV cohort. Current HIV research 2012; 10: 143-50.

39 Colonna M, Jonjic S, Watzl C. Natural killer cells: fighting viruses and much more. Nature immunology 2011; 12: 107-10.

40 Bielekova B, Richert N, Howard T, Blevins G, Markovic-Plese S, McCartin J, et al. Humanized anti-CD25 (daclizumab) inhibits disease activity in multiple sclerosis patients failing to respond to interferon beta. Proceedings of the National Academy of Sciences of the United States of America 2004; 101: 8705-8. 
41 Munschauer FE, Hartrich LA, Stewart CC, Jacobs L. Circulating natural killer cells but not cytotoxic $\mathrm{T}$ lymphocytes are reduced in patients with active relapsing multiple sclerosis and little clinical disability as compared to controls. Journal of neuroimmunology 1995; 62: 177-81.

42 Bielekova B, Catalfamo M. Regulatory CD56 (bright) natural killer cells mediate immunomodulatory effects of IL-2Ralpha-targeted therapy (daclizumab) in multiple sclerosis. Proceedings of the National Academy of Sciences of the United States of America 2006; 103: 5941-6.

43 Gregory AP, Dendrou C a, Attfield KE, Haghikia A, Xifara DK, Butter F, et al. TNF receptor 1 genetic risk mirrors outcome of anti-TNF therapy in multiple sclerosis. Nature 2012; 488: 508-11.

44 Lundström W, Highfill S, Walsh STR, Beq S, Morse E, Kockum I, et al. Soluble IL7Ra potentiates IL-7 bioactivity and promotes autoimmunity. Proceedings of the National Academy of Sciences of the United States of America 2013; 110: E1761-70.

45 Kappos L, Antel J, Comi G, Montalban X, O'Connor P, Polman CH, et al. Oral fingolimod (FTY720) for relapsing multiple sclerosis. The New England Journal of Medicine 2006; 355: 1124-40.

46 Hendrickson SL, Lautenberger JA, Chinn LW, Malasky M, Sezgin E, Kingsley LA, et al. Genetic Variants in Nuclear-Encoded Mitochondrial Genes Influence AIDS Progression. PLoS ONE 2010; 5: 8.

47 Campbell GR, Ohno N, Turnbull DM, Mahad DJ. Mitochondrial changes within axons in multiple sclerosis: an update. Current opinion in neurology 2012; 25: 221-30.

48 Michaelson MD, Mehler MF, Xu H, Gross RE, Kessler J a. Interleukin-7 is trophic for embryonic neurons and is expressed in developing brain. Developmental biology 1996; 179: 251-63.

49 Barrette B, Calvo E, Vallières N, Lacroix S. Transcriptional profiling of the injured sciatic nerve of mice carrying the Wld(S) mutant gene: identification of genes involved in neuroprotection, neuroinflammation, and nerve regeneration. Brain, behavior, and immunity 2010; 24: 1254-67.

50 McDonald WI, Compston a, Edan G, Goodkin D, Hartung HP, Lublin FD, et al. Recommended diagnostic criteria for multiple sclerosis: guidelines from the International Panel on the diagnosis of multiple sclerosis. Annals of neurology 2001; 50: 121-7.

51 Barrett J, Fry B, Maller J, Daly M. Haploview: analysis and visualization of LD and haplotype maps. Bioinformatics 2005; 21: 263-5.

52 Gentleman RC, Carey VJ, Bates DM, Bolstad B, Dettling M, Dudoit S, et al. Bioconductor: open software development for computational biology and bioinformatics. Genome Biology 2004; 5: R80.

53 Brunet J-P, Tamayo P, Golub TR, Mesirov JP. Metagenes and molecular pattern discovery using matrix factorization. Proceedings of the National Academy of Sciences of the United States of America 2004; 101: 4164-9. 
54 Gaujoux R, Seoighe C. A flexible R package for nonnegative matrix factorization. BMC Bioinformatics 2010; 11: 367. 


\section{Figure Legend}

Figure 1. Schematic diagram of the IL7RA sequence showing the four SNPs used to stratify the Hamburg cohort into four common haplotypes.

Figure 2. Haplotype dependent differences in IL-7RA mRNA and protein expression. (A) IL7RA mRNA expression from PBMCs of MS patients homozygous for haplotype 1-4 was determined by q-PCR and is shown as ratio of membrane bound to soluble IL7RA mRNA isoform (sample size: Hap1: 14, Hap2: 11, Hap3: 17, Hap4: 4). (B) Differences in IL-7RA serum concentration were analyzed by sandwich ELISA. The sIL-7RA serum levels were analyzed in samples from 32 haplotype 1, 25 haplotype 2, 30 haplotype 3, 4 haplotype 4 carrying homozygous and 11 heterozygous (for SNP rs6897932) MS patients. Results are presented as means \pm s.d.; * $P \leq 0.05$, ${ }^{* *} P \leq$ 0.005 and ${ }^{* *} \mathrm{P} \leq 0.001$.

Figure 3. Haplotype differences in IL-7RA surface expression. PBMCs of MS patients homozygous for either haplotype $1(n=15)$ or haplotype $3(n=14)$ were specifically stained for CD56 ${ }^{\text {bright }}$ cells and analyzed by flow cytometry. Results are presented as means \pm s.d.; * $\mathrm{P} \leq 0.05$.

Figure 4. Soluble IL-7RA is able to block IL-7 mediated signaling. IL-7 induced STAT5 phosphorylation in $\mathrm{CD}^{+} \mathrm{T}$ lymphocytes was measured by flow cytometry in vitro. (AD) Histograms show the STAT5 phosphorylation in $C D 4^{+} \mathrm{T}$ cells cultured in: 10 times concentrated HEK-cell supernatant, unstained unstimulated (dotted grey), unstimulated (dotted black), stimulated with 10-1000 pg/mL IL-7 (grey) and cultured in 10 times concentrated supernatant of a HEK-cell line producing soluble IL-7RA stimulated with $10-1000 \mathrm{pg} / \mathrm{mL}$ IL-7 (black). Representative results from three independent experiments are shown as mean values \pm s.d.; * $\mathrm{P} \leq 0.05$.

Figure 5. (A) Multidimensional scaling of individual response values (gene expression level after stimulation versus gene expression level in unstimulated cells). A subset (830 unique Ensembl gene IDs) corresponding to response values with low intra-group variability (lower third of median absolute difference per haplotype) was used. Color coding: haplotype 1, haplotype 2, and haplotype 3 are plotted as black, green, and red spheres, respectively. (B) Heatmap of $\mathrm{CD}^{+} \mathrm{T}$ cell responses to IL-7 stimulation for top-ranked metagenes. A subset of metagene-specific genes was selected from the top $10 \%$ contributing genes for each metagene and reconstructed values are shown (see Supplementary Material, Table S3 for raw data). Columns represent individual samples and rows the selected genes. Ordering of both samples and genes 
was obtained from hierarchical clustering showing dendrograms on top and left side, respectively. Colorcoding uses row-wise scaling to emphasize between-haplotype differences. (C) Interaction network of top metagenes as obtained from Reactome and STRING. Genes based on Reactome association alone (red labels) and additional genes from input list found associated via STRING (yellow nodes). For clarity, only direct interactions between genes within the cytokine signaling in immune system network are shown. 
Table 1. Allele and Genotype analysis of the four haplotype tagging SNPs rs1494555, rs6897932, rs987107 and rs987106 in 484 multiple sclerosis patients and $310 \mathrm{HDs}$ from Hamburg.

\begin{tabular}{|c|c|c|c|c|c|c|c|c|}
\hline SNP & Allele & $\begin{array}{c}\text { Frequency in } \\
\text { individuals with MS } \\
\end{array}$ & Frequency in controls & Genotype & $\begin{array}{c}\text { Frequency in individuals } \\
\text { with MS } \\
\end{array}$ & $\begin{array}{l}\text { Frequency in } \\
\text { controls } \\
\end{array}$ & $\begin{array}{c}\mathrm{p} \text {-value (dominat } \\
\text { coding) }\end{array}$ & $\begin{array}{c}\text { Odds ratio (95\% confidence } \\
\text { interval) }\end{array}$ \\
\hline \multirow[t]{3}{*}{ rs1494555 } & C & 0.35 & 0.34 & $\mathrm{C} / \mathrm{C}$ & 0.12 & 0.14 & & \\
\hline & $\mathrm{T}$ & 0.65 & 0.66 & $\mathrm{C} / \mathrm{T}$ & 0.45 & 0.40 & & \\
\hline & & & & $\mathrm{T} / \mathrm{T}$ & 0.43 & 0.46 & 0.38 & $0.88(0.66-1.17)$ \\
\hline \multirow[t]{3}{*}{ rs6897932 } & $\mathrm{T}$ & 0.24 & 0.27 & $\mathrm{~T} / \mathrm{T}$ & 0.07 & 0.05 & & \\
\hline & C & 0.76 & 0.73 & $\mathrm{C} / \mathrm{T}$ & 0.33 & 0.43 & & \\
\hline & & & & $\mathrm{C} / \mathrm{C}$ & 0.60 & 0.52 & 0.03 & $1.38(1.04-1.84)$ \\
\hline \multirow[t]{3}{*}{ rs987107 } & $\mathrm{T}$ & 0.30 & 0.26 & $T / T$ & 0.10 & 0.06 & & \\
\hline & C & 0.70 & 0.74 & $\mathrm{C} / \mathrm{T}$ & 0.40 & 0.39 & & \\
\hline & & & & $\mathrm{C} / \mathrm{C}$ & 0.50 & 0.55 & 0.28 & $0.85(0.64-1.13)$ \\
\hline \multirow[t]{3}{*}{ rs987106 } & $\mathrm{T}$ & 0.46 & 0.48 & $T / T$ & 0.24 & 0.23 & & \\
\hline & $A$ & 0.54 & 0.52 & $\mathrm{~A} / \mathrm{T}$ & 0.45 & 0.50 & & \\
\hline & & & & $\mathrm{A} / \mathrm{A}$ & 0.31 & 0.27 & 0.30 & $1.19(0.87-1.63)$ \\
\hline
\end{tabular}


Table 2. Haplotype analysis of IL-7RA in 484 MS patients and 310 HDs from northern Germany using four adjacent SNPs that distinguish all common haplotypes

\begin{tabular}{lccccc}
\hline & Haplotype & Frequency & $\begin{array}{c}\text { Frequency in } \\
\text { individuals with } \\
\text { MS }\end{array}$ & $\begin{array}{c}\text { Frequency in } \\
\text { controls }\end{array}$ & p-value \\
\hline \hline $\begin{array}{l}\text { Haplotype 1 } \\
\text { (risk Hap) }\end{array}$ & CCCT & 0.34 & 0.35 & 0.34 & 0.78 \\
Haplotype 2 & TCTA & 0.28 & 0.30 & 0.26 & 0.09 \\
Haplotype 3 & TTCA & 0.25 & 0.24 & 0.27 & 0.20 \\
(protective Hap) & TCCT & 0.12 & 0.12 & 0.14 & 0.24 \\
\hline
\end{tabular}


Table 3. Gene expression differences in $\mathrm{CD}^{+} \mathrm{T}$ cells of MS individuals homozygous for one of three haplotypes (Hap1, Hap2, Hap3). Only genes whose expression levels varies maximally between haplotypes (top $0.1 \%$ divergent gene expression from F-test statistics, corresponding to $p$-value $\leq 1.8 \mathrm{E}-3$ ) and show the largest distances (top $1 \%$ of effect values) are shown.

\begin{tabular}{cccccc}
\hline Ensembl ID & Symbol & p-value & $\begin{array}{c}\text { LogFC Hap1 vs } \\
\text { Hap3 }\end{array}$ & $\begin{array}{c}\text { LogFC Hap1 vs } \\
\text { Hap2 }\end{array}$ & $\begin{array}{c}\text { LogFC Hap3 vs } \\
\text { Hap2 }\end{array}$ \\
\hline \hline ENSG00000202089 & U6 snRNA & $8.26 \mathrm{E}-04$ & -0.91 & -2.38 & -1.47 \\
ENSG00000212527 & RN5S63 & $1.47 \mathrm{E}-04$ & 0.99 & -0.24 & -1.23 \\
ENSG00000200796 & U6 snRNA & $5.39 \mathrm{E}-04$ & -0.50 & -1.04 & -0.54 \\
ENSG00000187172 & BAGE2 & $1.43 \mathrm{E}-03$ & -0.64 & 0.34 & 0.97 \\
ENSG00000206826 & U6 snRNA & $2.30 \mathrm{E}-06$ & -0.10 & -0.94 & -0.84 \\
ENSG00000201813 & U6 snRNA & $9.35 \mathrm{E}-05$ & -0.17 & -0.88 & -0.71 \\
ENSG00000120322 & PCDHB8 & $1.96 \mathrm{E}-04$ & -0.17 & -0.87 & -0.70 \\
ENSG00000201088 & Y RNA & $8.18 \mathrm{E}-04$ & -0.22 & -0.84 & -0.63 \\
ENSG00000125144 & MT1G & $7.88 \mathrm{E}-04$ & -0.09 & 0.70 & 0.79 \\
ENSG00000201065 & U6 snRNA & $7.17 \mathrm{E}-04$ & -0.25 & -0.79 & -0.53 \\
ENSG00000252574 & RNU5B-6P & $2.18 \mathrm{E}-05$ & -0.02 & -0.70 & -0.68 \\
\hline
\end{tabular}


Table 4. Genes induced by stimulating isolated $\mathrm{CD}^{+} \mathrm{T}$ cells with IL-7. Top $1 \%$ of significantly regulated genes corresponding to adjusted p-value $\leq 1.6 \mathrm{E}-5$ sorted by absolute log fold change (10 entries each from strongly up- and down-regulated genes, respectively). Expression differences were calculated by combining samples according to stimulus (either unstimulated or stimulated) irrespectively of their haplotype assignment.

\begin{tabular}{cccc}
\hline Ensembl ID & Symbol & adj. p-value & logFC \\
\hline \hline ENSG00000114737 & CISH & $2.66 \mathrm{E}-11$ & 2.69 \\
ENSG00000105810 & CDK6 & $3.68 \mathrm{E}-10$ & 2.61 \\
ENSG00000125384 & PTGER2 & $3.36 \mathrm{E}-09$ & 2.55 \\
ENSG00000137265 & IRF4 & $3.02 \mathrm{E}-10$ & 2.48 \\
ENSG00000171791 & BCL2 & $8.51 \mathrm{E}-12$ & 2.40 \\
ENSG00000079385 & CEACAM1 & $2.45 \mathrm{E}-09$ & 2.33 \\
ENSG00000067113 & PPAP2A & $1.25 \mathrm{E}-09$ & 1.56 \\
ENSG00000157800 & SLC37A3 & $3.36 \mathrm{E}-09$ & 1.23 \\
ENSG00000074800 & ENO1 & $3.03 \mathrm{E}-09$ & 1.16 \\
ENSG00000171793 & CTPS & $3.36 \mathrm{E}-09$ & 1.13 \\
ENSG00000123329 & ARHGAP9 & $1.91 \mathrm{E}-06$ & -0.62 \\
ENSG00000165071 & TMEM71 & $4.86 \mathrm{E}-06$ & -0.74 \\
ENSG00000137642 & SORL1 & $2.66 \mathrm{E}-06$ & -0.83 \\
ENSG00000167261 & DPEP2 & $3.04 \mathrm{E}-06$ & -0.87 \\
ENSG00000070731 & ST6GALNAC2 & $7.64 \mathrm{E}-06$ & -0.87 \\
ENSG00000150054 & MPP7 & $3.06 \mathrm{E}-06$ & -0.90 \\
ENSG00000106560 & GIMAP2 & $7.11 \mathrm{E}-06$ & -0.91 \\
ENSG00000162894 & FAIM3 & $8.73 \mathrm{E}-08$ & -1.12 \\
ENSG00000168685 & IL7R & $3.95 \mathrm{E}-07$ & -1.40 \\
ENSG00000176928 & GCNT4 & $1.42 \mathrm{E}-06$ & -1.47 \\
\hline
\end{tabular}

